\title{
Thermal production, protection, and heat exchange of quantum coherences
}

\author{
B. Çakmak, ${ }^{*}$ A. Manatuly, and Ö. E. Müstecaplığlu \\ Department of Physics, Koç University, Istanbul, Sartyer 34450, Turkey
}

(Received 8 June 2017; published 22 September 2017)

\begin{abstract}
We consider finite-sized atomic systems with varying number of particles which have dipolar interactions among them and are also under the collective driving and dissipative effect of a thermal photon environment. Focusing on the simple case of two atoms, we investigate the impact of different parameters of the model on the coherence contained in the system. We observe that, even though the system is initialized in a completely incoherent state, it evolves to a state with a finite amount of coherence and preserves that coherence in the long-time limit in the presence of thermal photons. We propose a scheme to utilize the created coherence in order to change the thermal state of a single two-level atom by having it repeatedly interact with a coherent atomic beam. Finally, we discuss the scaling of coherence as a function of the number of particles in our system up to $N=7$.
\end{abstract}

DOI: 10.1103/PhysRevA.96.032117

\section{INTRODUCTION}

The vast majority of actual quantum systems are not isolated and are in direct or indirect contact with their surrounding environments. The field of the theory of open quantum systems deals with the problem of understanding the physics behind this mechanism and is a well-established topic [1-3]. In general, interaction with the environment results in the loss of all quantum properties of the system, but most important of all is coherence.

Many peculiarities of quantum mechanics can be traced back to the wave-particle duality property of quantum particles. This dual behavior allows us to describe these particles as waves and put them in a coherent superposition of two (or more) possible states that they are allowed to occupy. The presence of such coherent superposition states actually is one of the main differences between quantum mechanics and classical mechanics. Despite its importance and many different manifestations, the genuine framework of characterization and quantification of coherence in an arbitrary quantum system was introduced only very recently [4-7]. The framework is formalized in [4] by introducing a set of physically motivated conditions for a proper measure of coherence. Quantification of quantum coherence has attracted a lot of attention both at the fundamental level and about its applications in quantum critical, open, and biological systems [8-29].

Quantum coherence is typically considered to be a resource for quantum information devices [17-25]. More recently, it was understood that it can also be used as a "fuel" for quantum heat engines (QHEs) [25,30-36]. Such a profound QHE, which can convert quantum coherence to useful work, can be practically appealing only if the abundant coherence is produced and protected either naturally or by energetically cheap artificial methods. Moreover, it is necessary to be able to have a scheme that can harvest stored coherences as heat to produce work in a genuine heat engine cycle. Early studies, focusing on entanglement of a pair of two-level atoms, suggested that a promising route towards natural and

\footnotetext{
*bcakmak@ku.edu.tr
}

long-lived quantum coherence could be achieved by simply using a thermal environment [37-42]. However, such schemes yield too-small quantum coherence. How to scale them up and how to convert them back to heat for the realization of profound QHEs remain as open questions.

In this paper, we propose that an ensemble of two-level atoms can produce a large amount of many-body quantum coherence by collective coupling to a thermal environment. We find that the coherence exhibits a superlinear scaling with the number of ensemble atoms. In addition, we propose a scheme to harvest such thermally generated quantum coherence back as heat. We find that a single two-level atom can be used as the working medium to harvest the coherences by randomly and repeatedly making it interact with similarly prepared coherent atomic clusters (pairs). The working atom reaches a steady state that can be described by a thermal equilibrium state whose temperature depends on the coherence. This is in fact a generalization of a well-known route to thermalization by collision models $[43,44]$, as well as the photo-Carnot engine in which the working fluid is the micromaser cavity field [30]. The intriguing point is that only certain coherences can be produced by collective heating and only those that can be converted back to heat. These coherences share the characteristic property of belonging to the energy degenerate subspaces (Dicke-type states or Wigner- $j$ matrix blocks in the computational basis) which was recently classified as heat-exchange coherence [32]. Collective heating can "charge" such "flammable" coherences even if they are not present initially and preserve them in steady state so that they can be "discharged" back to heat by the initiation of a harvesting scheme (cf. Fig. 1).

The organization of the paper is as follows. We first describe our model system in Sec. II. We then focus on the case of a pair of two-level atoms and present key results on the quantum coherence generation and protection in a thermal environment in Sec. III. Sections III A and III B focus on the effects of the environment temperature and the dipolar coupling between the atoms, respectively. Section III C introduces our proposed scheme to harvest the thermally produced and stored coherences back as heat using a single two-level atom. We generalize our results to the case of multiple atoms in Sec. IV. We conclude in Sec. V. 


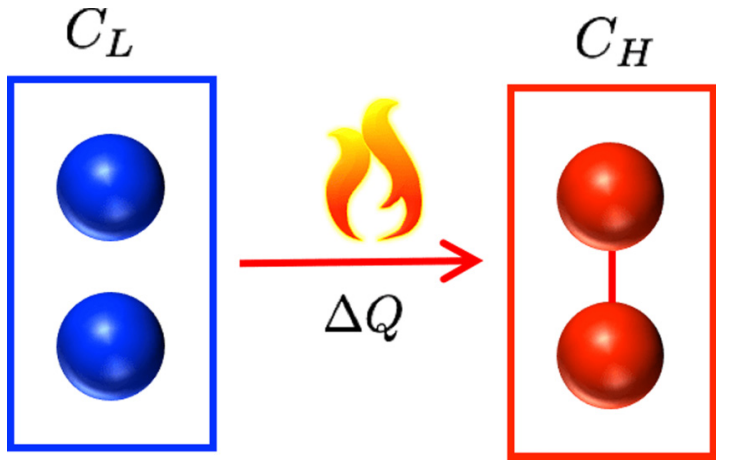

(a)

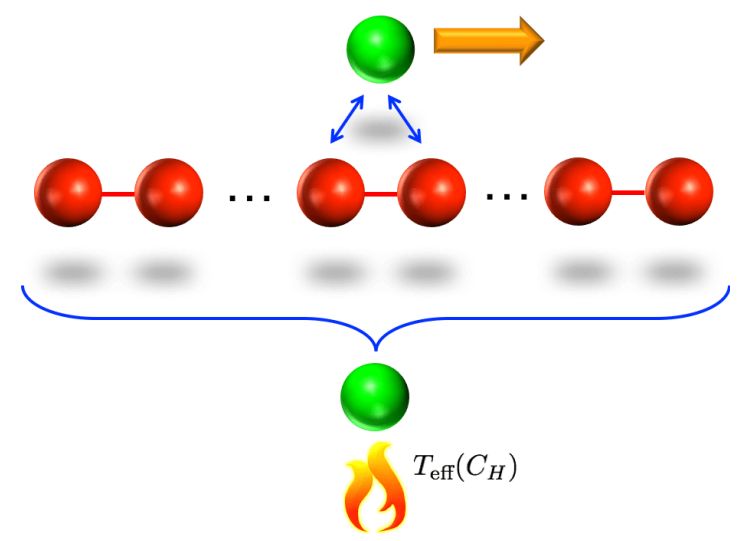

(b)

FIG. 1. Schematic view of (a) creation and (b) harvesting of coherences in our model. A pair of two-level atoms initially in a state with coherence $C_{L}$ can be transformed into another state with higher coherence $C_{H}>C_{L}$ by collective interaction with a thermal bath. The energetic cost of the generation of coherences is paid by the "natural" heat $\Delta Q$ withdrawn from the thermal bath. Some amount of this energy can be harvested back by a repeated interaction method where a single two-level atom is coupled sequentially with a pair of atoms with coherence $C_{H}$. The coupling happens at random times and the atom reaches a steady state eventually that can be described by a thermal state with an effective temperature $T_{\text {eff }}$ that can be controlled by the $C_{H}$ of the subenvironment atomic pairs. (a) Heat is used to produce ("charge") coherences. (b) Coherences are harvested back ("discharged") as heat.

\section{MODEL}

The internal Hamiltonian of the atoms is given by $H_{s}=$ $\left(\hbar \omega_{0} / 2\right) \sum_{i=1}^{N} \sigma_{i}^{z}$, where $\sigma_{i}^{z}=\left|e_{i}\right\rangle\left\langle e_{i}|-| g_{i}\right\rangle\left\langle g_{i}\right|$ is the Pauli $z$ matrix for the $i$ th atom and $\omega_{0}$ is the transition frequency of the atom. For simplicity, we assume that all of the atoms in our sample act like point dipoles and are polarized such that they all have the same dipole moment $\mathbf{d}_{\text {eg }}^{\mathbf{i}}=\mathbf{d}_{\mathrm{eg}}=\langle e|\mathbf{d}| g\rangle$. In the interaction picture associated with $H_{s}$, the master equation governing the dynamics of the atomic system is given by

$$
\frac{d \rho}{d t}=-\frac{i}{\hbar}\left[H_{d}, \rho\right]+\mathcal{D}_{-}(\rho)+\mathcal{D}_{+}(\rho)=\mathcal{L}(\rho),
$$

where the first term accounts for the unitary dipole-dipole interaction. The second and third terms describe the spontaneous and thermally induced emission (dissipation) and thermally induced absorption (driving) processes, respectively, whose explicit forms are as follows:

$$
\mathcal{D}_{-}(\rho)=\sum_{i, j=1}^{N} \gamma_{i j}(\bar{n}+1)\left(\sigma_{j}^{-} \rho \sigma_{i}^{+}-\frac{1}{2}\left\{\sigma_{i}^{+} \sigma_{j}^{-}, \rho\right\}\right)
$$

and

$$
\mathcal{D}_{+}(\rho)=\sum_{i, j=1}^{N} \gamma_{i j} \bar{n}\left(\sigma_{j}^{+} \rho \sigma_{i}^{-}-\frac{1}{2}\left\{\sigma_{i}^{-} \sigma_{j}^{+}, \rho\right\}\right) .
$$

In the equations above, $\sigma_{i}^{+}=\left|e_{i}\right\rangle\left\langle g_{i}\right|$ and $\sigma_{i}^{-}=\left|g_{i}\right\rangle\left\langle e_{i}\right|$ are the raising and lowering operators, respectively, for the $i$ th atom, and $\bar{n}=\left(\exp \left(\beta \hbar \omega_{0}\right)-1\right)^{-1}$ is the mean number of thermal photons at the transition frequency of the atom at an inverse temperature $\beta . H_{d}=\hbar \sum_{i \neq j} f_{i j} \sigma_{i}^{+} \sigma_{j}^{-}$is the dipole-dipole coupling Hamiltonian between the atoms in the considered system. The dipolar interaction strength $f_{i j}$ and the dissipation and driving rates $\gamma_{i j}$ are given as [45-47]

$$
\begin{aligned}
f_{i j}= & \frac{3 \gamma_{0}}{4}\left[\left(1-3 \cos ^{2} \alpha_{i j}\right)\left(\frac{\sin \xi_{i j}}{\xi_{i j}^{2}}+\frac{\cos \xi_{i j}}{\xi_{i j}^{3}}\right)\right. \\
& \left.-\left(1-\cos ^{2} \alpha_{i j}\right) \frac{\cos \xi_{i j}}{\xi_{i j}}\right]
\end{aligned}
$$

and

$$
\begin{aligned}
\gamma_{i j}= & \frac{3 \gamma_{0}}{2}\left[\left(1-3 \cos ^{2} \alpha_{i j}\right)\left(\frac{\cos \xi_{i j}}{\xi_{i j}^{2}}-\frac{\sin \xi_{i j}}{\xi_{i j}^{3}}\right)\right. \\
& \left.+\left(1-\cos ^{2} \alpha_{i j}\right) \frac{\sin \xi_{i j}}{\xi_{i j}}\right] .
\end{aligned}
$$

Here, $\gamma_{0}=\left(\omega_{0}^{3} d_{e g}^{2}\right) /\left(3 \pi \hbar \epsilon_{0} c^{3}\right)$ is the single-atom spontaneous emission rate, $\xi_{i j}=k_{0} r_{i j}$ is a dimensionless parameter characterizing the distance between the particles with $k_{0}=\omega_{0} / c$, and $r_{i j}=\left|\mathbf{r}_{\mathbf{i j}}\right|=\left|\mathbf{r}_{\mathbf{i}}-\mathbf{r}_{\mathbf{j}}\right|$ gives the relative positions of the $i$ th and $j$ th atoms. Finally, $\alpha_{i j}$ is the angle between $\mathbf{r}_{\mathbf{i j}}$ and $\mathbf{d}_{\mathbf{e g}}$.

The model admits two different regimes depending on the spatial distance of the atoms inside the ensemble. On one hand, we have the $\xi_{i j} \gg 1$ limit describing that every atom is significantly distant from each other, which results in the approximate model parameters $f \approx 0$ and $\gamma_{i j} \approx \gamma_{0} \delta_{i j}$ for all $i, j$. In this regime we have no collective effects; every particle behaves independently. On the other hand, in the complete opposite limit of $\xi_{i j} \ll 1$ where $f_{i j} \approx\left(3 \gamma_{0}(1-\right.$ $\left.\left.3 \cos ^{2} \alpha_{i j}\right)\right) / 4 \xi_{i j}^{3}$ and $\gamma_{i j} \approx \gamma_{0}$ for all $i, j$, we are in the regime where collective effects play an important role.

\section{DYNAMICS OF COHERENCE}

To begin with, we introduce the coherence measure that we are going to utilize to determine the amount of coherence in our system. It is called the $l_{1}$ norm of coherence and is just given by the sum of the absolute values of the off-diagonal elements in the density matrix of a given quantum system [4]

$$
C_{l_{1}}(t)=\sum_{i \neq j}\left|\rho_{i, j}(t)\right|
$$

$C_{l_{1}}$ is meaningful only if a reference basis for the density matrix is set and, in what follows, we fix our reference basis to be $\left\{\left|e_{1} e_{2} \cdots e_{n-1} e_{n}\right\rangle,\left|e_{1} e_{2} \cdots e_{n-1} g_{n}\right\rangle,\left|e_{1} e_{2} \cdots g_{n-1} e_{n}\right\rangle, \ldots\right.$, 
$\left.\left|g_{1} g_{2} \cdots g_{n-1} g_{n}\right\rangle\right\}$, namely, a excitation or computational basis.

We may now proceed to analyze the time evolution of the $l_{1}$ norm under the dynamics dictated by the master equation of our physical model, Eq. (1), for two atoms. We specifically concentrate on the case of $N=2$, since it is the simplest ground in order to analyze the impact of different parameters in the model on the time evolution of the atoms.

As previously mentioned, the dynamical model we consider here has two different regimes depending on the separation between the atoms. In the limit of $\xi_{i j} \gg 1$ for which the atoms are far apart from each other, we do not observe any interesting phenomena in the course of dynamics. Initial states that have no coherence do not accumulate any and the ones that have coherence lose it monotonically in finite time. In other words, individual coupling of atoms to the environment generates no coherence and destroys the initially present amount. Therefore, we assume that the atoms in our system are spatially very close, corresponding to the $\xi_{i j} \ll 1$ limit where the collective effects are pronounced. Throughout this paper we only consider identical dipole-dipole interactions between the atoms: $f_{i j}=$ $f_{0}$. Furthermore, we make an implicit assumption that the mean number of photons, $\bar{n}$, appearing in Eq. (1) is actually the mean number of photons that are at the transition frequency of the atoms, $\omega_{0}$, at a given temperature.

Before moving on to the multiatom cases, we first consider the dynamics of coherence for a cluster constituted by two atoms in order to demonstrate interplay between the two main parameters in the system: the mean number of photons in the environment, $n$, and the dipole-dipole interaction constant $f_{0}$. The conclusions we draw about the effect of these parameters also apply to larger atomic clusters.

\section{A. Impact of mean number of thermal photons}

The discussion on the effect of the mean number of photons in the environment surrounding the atoms is a bit more complicated to treat, even partially, for the most general initial state of two atoms. Therefore, we need to choose an initial state and we have decided that initiating the dynamics from the ground state would generate the most suitable scenario to demonstrate the effect of thermal photons on coherence. If we take the initial state of the ensemble as the ground state of both atoms, $\rho(t=0)=\left|g_{1} g_{2}\right\rangle\left\langle g_{1} g_{2}\right|$, and evolve it according to Eq. (1), we can calculate $\rho(t)$. We find that it is always in the form of a symmetric $X$-type (Dicke-type) state with only two nonzero coherences lying in the central block. The coherences are always positive. $C_{l_{1}}$ as defined in Eq. (4) can be evaluated analytically (see Fig. 2). The result is independent of the dipolar interactions and given by

$$
\begin{aligned}
C_{l_{1}}(t)= & \frac{\bar{n}(\bar{n}+1)}{3 \bar{n}(\bar{n}+1)+1} \\
& -\frac{\bar{n} e^{-a t}\left[(\bar{n}+1) \sqrt{\bar{n}(\bar{n}+1)} \cosh (b t)-\bar{n}^{2} \sinh (b t)\right]}{[3 \bar{n}(\bar{n}+1)+1] \sqrt{\bar{n}(\bar{n}+1)}},
\end{aligned}
$$

where $a=2 \gamma_{0}(2 \bar{n}+1)$ and $b=2 \gamma_{0} \sqrt{\bar{n}(\bar{n}+1)}$. We now analyze the results of different limits admitted by the above equation.

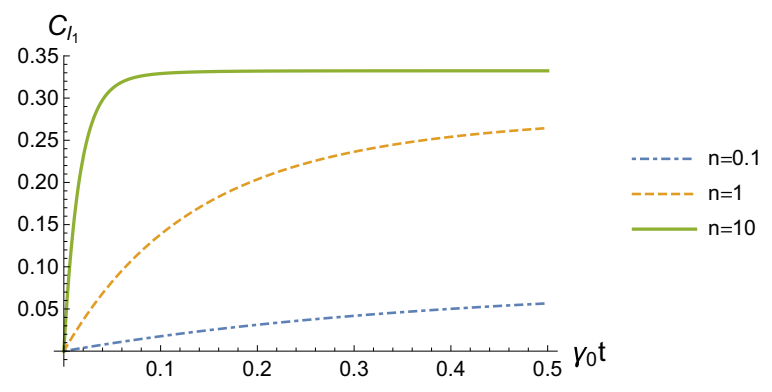

FIG. 2. $C_{l_{1}}$ as a function of scaled time $\gamma_{0} t$ for different mean number of photons, $\bar{n}$, in the environment for the two atoms initially in the ground state.

To begin with, when there are no thermally induced processes, i.e., $\bar{n}=0, C_{l_{1}}$ remains zero, independent of time. Since in the absence of thermal environmental photons only a spontaneous decay mechanism is present, an atomic system initiated in its ground state does not change during such dynamics. However, as soon as we have a finite-temperature environment embodied by thermal photons, $\bar{n} \neq 0$, we begin to have nonzero coherence in the state of the system. As $t \rightarrow \infty$, the second term on the right-hand side of Eq. (5) goes to zero, leaving the first term unaffected, which implies a time-invariant coherence in the system. In addition, in the case of high-temperature environments where the mean number of photons is high, $n \gg 1, C_{l_{1}}$ converges to the value $1 / 3$.

The mechanism behind the creation of coherence in the atomic ensemble is the thermal drive term in our physical model and this term is present only when we have $n \neq 0$. The very presence of thermal photons in the environment surrounding our system makes it possible for an atom to absorb that photon and make a transition to the excited state. Since we assume that the atoms in the ensemble are very close, they can be treated as though they are indistinguishable. Therefore, it is not possible to identify the atom that absorbed the photon and ended up in the excited state, which puts our system in a superposition state in the single (or more) excitation Hilbert space. However, the dissipation in the system, partially caused also by the same environment of thermal photons, opens up a channel that causes decays to the ground state, leading to loss of some of these created excitations. Nevertheless, in the long-time limit, we see that our atomic ensemble comes to an equilibrium state, due to the trade-off between the loss and drive mechanisms, with a finite amount of coherence.

\section{B. Impact of dipolar interactions}

In order to investigate the impact of dipole-dipole interactions, we first determine the initial states whose evolution is influenced by $H_{d}$. For that aim, we have assumed that the system is disconnected from the outside environment, i.e., $\gamma_{i j}=0$, and it only evolves unitarily as determined by the first term in Eq. (1). The evolution of elements of the density matrix are presented in Eq. (A1), in Appendix A. By inspection one can conclude that there are some initial states which are indifferent to the presence of dipolar interactions during the time evolution. These initial density matrices have $\rho_{11}, \rho_{14}$ (and naturally $\rho_{41}$ ), and/or $\rho_{44}$ as their only nonzero 

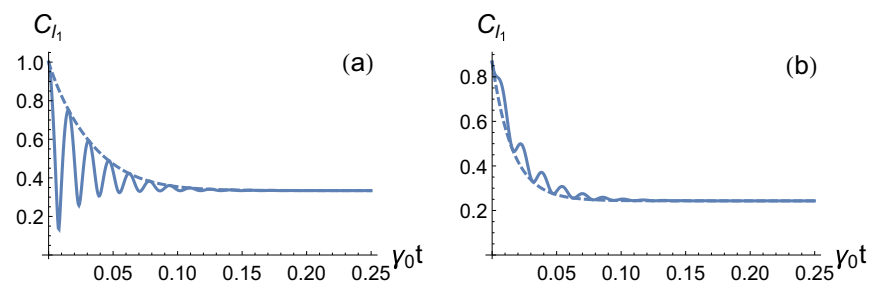

FIG. 3. Dynamics of $C_{l_{1}}$ for the initial state (a) $|\psi(0)\rangle=(|g e\rangle+$ $i|e g\rangle) / \sqrt{2}$ and (b) $|\psi(0)\rangle=(\sqrt{3}|g e\rangle+|e g\rangle) / 2$. Solid and dashed lines represent $f_{0} / \gamma_{0}=10^{2}$ and $f_{0} / \gamma_{0}=1$ cases, respectively, with $\bar{n}=10$. Time is dimensionless and scaled with $\gamma_{0}$.

elements, or satisfying the condition $\rho_{22}=\rho_{33}$ together with $\rho_{23} \in$ Re. Initial states lying outside the mentioned cases are affected by dipolar interactions and the effect is reflected to the dynamics of coherence as sinusoidal oscillations. Since the system evolution is unitary under $H_{d}$, such a behavior is natural. It is important to note that, when we consider the interactions with the environment, Eq. (A1) describing the evolution of the density matrix elements will surely change and the effect of coherent dipole interactions will be suppressed due to open system dynamics. However, the dipolar interactions will affect the same density matrix elements apart from the cases outlined above; thus our conclusions above still hold true in the presence of interaction with the environment.

Figure 3 exemplifies the effect of dipole-dipole interactions for two different initial states for which the interaction does have an impact. It is possible to conclude that, in the presence of dissipation and drive, dipole interactions only have appreciable effects on the dynamics of coherence in short times: the value of coherence in the long-time limit does not depend on it. Moreover, to be able to see the effects in short times, the system must be in the regime $f_{0} / \gamma_{0}>1$ with $\bar{n}=10$, which are two competing energy scales in the dynamics. Even having $f_{0} / \gamma_{0} \approx 1$ is not enough to see an observable difference in the behavior of coherence.

\section{Harvesting the coherences}

In the previous sections we have seen that collective coupling of a pair of two-level atoms to a heat bath allows for generation of certain coherences in the two-atom density matrix. In this section we explore a scheme to harvest these coherences back as heat. For that aim, we consider a beam of atomic pairs carrying such coherences and assume that they interact with a two-level atom at random time intervals. The total Hamiltonian describing the system can be written as follows:

$$
H=H_{\mathrm{q}}+H_{\mathrm{b}}+H_{\text {int }},
$$

where the single atom, an atom pair in the beam, and the interaction Hamiltonians are respectively given by

$$
\begin{gathered}
H_{\mathrm{q}}=\frac{\hbar \omega_{0}}{2} \sigma_{0}^{z}, \\
H_{\mathrm{b}}=\frac{\hbar \omega_{0}}{2} \sum_{i=1}^{2} \sigma_{i}^{z}, \\
H_{\mathrm{int}}=\hbar g \sum_{i=1}^{2}\left(\sigma_{i}^{+} \sigma_{0}^{-}+\sigma_{i}^{-} \sigma_{0}^{+}\right) .
\end{gathered}
$$

Here $\omega_{0}$ is the transition frequency of the atoms, which are taken to be identical. The interaction coefficient is denoted by $g$.

The atomic pairs arrive randomly at a rate $p$, and the interaction time $\tau$ is assumed to be short such that the condition $g \tau \ll 1$ is satisfied. In the interaction picture, the timeevolution operator is given by $U(\tau)=\exp \left(-i H_{\text {int }} \tau\right)$ whose exact expression, up to second order in $(g \tau)^{2}$, is given in Appendix B. The total density matrix of the system before each interaction is the product of the constituent density matrices: $\rho(t)=\rho_{b}(t) \otimes \rho_{q}(t)$. Then, the master equation of the qubit can be written as

$$
\dot{\rho}_{q}(t)=p\left[\sum_{i, j=1}^{N} a_{i j} \sum_{n=1}^{N} U_{n i}(\tau) \rho_{q}(t)\left[U_{n j}(\tau)\right]^{\dagger}-\rho_{q}(t)\right],
$$

where $a_{i j}$ are the density matrix elements of an atomic pair in the beam $\rho_{b}(t) . U_{n i(j)}$ are the matrix elements of the time-evolution operator $U(\tau)$. It is important to note that these matrix elements are actually operators in the single-qubit Hilbert space. Expanding $U_{n i}$ and $U_{n j}$, we obtain the master equation in the Lindblad form:

$$
\dot{\rho}_{q}=-i\left[H_{\mathrm{eff}}, \rho_{q}\right]+\mathcal{L}_{s} \rho_{q}+\mathcal{L} \rho_{q} .
$$

The Hamiltonian $H_{\text {eff }}$ describes a coherent-drive term on the atom:

$$
H_{\mathrm{eff}}=p g \tau\left(\lambda \sigma^{+}+\lambda^{*} \sigma^{-}\right) .
$$

The Lindbladian $\mathcal{L}_{s}$ describes a squeezed reservoir effect on the atom and is expressed as

$$
\begin{aligned}
\mathcal{L}_{s} \rho_{q} & =2 \mu\left(\epsilon \sigma^{+} \rho_{q} \sigma^{+}+\epsilon^{*} \sigma^{-} \rho_{q} \sigma^{-}\right) \\
& =2 \mu\left(\epsilon \mathcal{L}_{s}^{e} \rho_{q}+\epsilon^{*} \mathcal{L}_{s}^{d} \rho_{q}\right)
\end{aligned}
$$

where $\mu=p(g \tau)^{2}$. The Lindbladian $\mathcal{L} \rho_{q}$ is expressed by

$$
\mathcal{L} \rho_{q}=\mu\left(\frac{r_{e}}{2} \mathcal{L}_{e} \rho_{q}+\frac{r_{d}}{2} \mathcal{L}_{d} \rho_{q}\right)
$$

where excitation and deexcitation of the atom are described by

$$
\begin{aligned}
& \mathcal{L}_{e} \rho_{q}=2 \sigma^{+} \rho_{q} \sigma^{-}-\sigma^{-} \sigma^{+} \rho_{q}-\rho_{q} \sigma^{-} \sigma^{+}, \\
& \mathcal{L}_{d} \rho_{q}=2 \sigma^{-} \rho_{q} \sigma^{+}-\sigma^{+} \sigma^{-} \rho_{q}-\rho_{q} \sigma^{+} \sigma^{-} .
\end{aligned}
$$

Table I shows that coherences in the density matrix of the atomic pair disjointly determine the contributions of the

TABLE I. The coefficients of the Lindbladians in the master equation.

\begin{tabular}{lc}
\hline \hline$r_{e}$ & $2 a_{11}+a_{22}+a_{23}+a_{32}+a_{33}$ \\
$r_{d}$ & $2 a_{44}+a_{22}+a_{23}+a_{32}+a_{33}$ \\
$\lambda$ & $a_{12}+a_{13}+a_{24}+a_{34}$ \\
$\epsilon$ & $a_{14}$ \\
\hline \hline
\end{tabular}


processes in the master equation. If the state of the atomic pairs is set so that $\lambda=0$, the master equation becomes

$$
\dot{\rho}_{q}=\mathcal{L}_{s} \rho_{q}+\mathcal{L} \rho_{q} .
$$

By expressing $N=r_{e} /\left(r_{d}-r_{e}\right), \gamma=\mu\left(r_{d}-r_{e}\right)$, and $M e^{i \phi}=$ $-2 \epsilon \mu / \gamma$, we can rewrite the equation as follows:

$$
\begin{aligned}
\dot{\rho}_{q}= & \frac{1}{2} \gamma(N+1)\left(2 \sigma^{-} \rho_{q} \sigma^{+}-\sigma^{+} \sigma^{-} \rho_{q}-\rho_{q} \sigma^{+} \sigma^{-}\right) \\
& +\frac{1}{2} \gamma N\left(2 \sigma^{+} \rho_{q} \sigma^{-}-\sigma^{-} \sigma^{+} \rho_{q}-\rho_{q} \sigma^{-} \sigma^{+}\right) \\
& -\gamma M e^{i \phi} \sigma^{+} \rho_{q} \sigma^{+}-\gamma M e^{-i \phi} \sigma^{-} \rho_{q} \sigma^{-} .
\end{aligned}
$$

The master equation is the same as that of the two-level atom subjected to the squeezed thermal bath, with the Bloch equations [48]

$$
\begin{aligned}
\left\langle\dot{\sigma}_{x}\right\rangle= & -\frac{\gamma}{2}\left(2 N+M+M^{*}+1\right)\left\langle\sigma_{x}\right\rangle \\
& -\frac{i \gamma}{2}\left(M-M^{*}\right)\left\langle\sigma_{y}\right\rangle+\frac{1}{2} \operatorname{ipg} \tau\left(\lambda-\lambda^{*}\right)\left\langle\sigma_{z}\right\rangle, \\
\left\langle\dot{\sigma_{y}}\right\rangle= & -\frac{\gamma}{2}\left(2 N+M+M^{*}+1\right)\left\langle\sigma_{y}\right\rangle \\
& -\frac{i \gamma}{2}\left(M-M^{*}\right)\left\langle\sigma_{x}\right\rangle-\frac{1}{2} \operatorname{pg} \tau\left(\lambda+\lambda^{*}\right)\left\langle\sigma_{z}\right\rangle, \\
\left\langle\dot{\sigma}_{z}\right\rangle= & -\gamma\left[(2 N+1)\left\langle\sigma_{z}\right\rangle+1\right] \\
& -2 i p g \tau\left[\left(\lambda-\lambda^{*}\right)\left\langle\sigma_{x}\right\rangle+i\left(\lambda+\lambda^{*}\right)\left\langle\sigma_{y}\right\rangle\right],
\end{aligned}
$$

where $\gamma$ corresponds to the vacuum spontaneous emission rate.

The nonunitary part of the master equation in fact could be transformed into a sum of two $\mathcal{L}_{1}$ and $\mathcal{L}_{2}$ dissipators in Lindblad form. We can transform them into two dissipators using $R_{1}$ and $R_{2}$ [49]:

$$
\begin{aligned}
\dot{\rho}_{q} & =-i\left[H_{\mathrm{tot}}, \rho_{q}\right]+\mathcal{L}_{1}+\mathcal{L}_{2} \\
& =-i\left[H_{\mathrm{tot}}, \rho_{q}\right]+\sum_{i=1}^{2}\left(2 R_{i} \rho_{q} R_{i}^{\dagger}-R_{i}^{\dagger} R_{i} \rho_{q}-\rho_{q} R_{i}^{\dagger} R_{i}\right),
\end{aligned}
$$

where

$$
\begin{aligned}
R_{1} & =\sqrt{\frac{\gamma\left(N_{\mathrm{th}}+1\right)}{2}} R, \\
R_{2} & =\sqrt{\frac{\gamma N_{\mathrm{th}}}{2}} R^{\dagger}, \\
R & =\sigma^{-} \cosh (r)+e^{i \phi} \sigma^{+} \sinh (r) .
\end{aligned}
$$

The master equation is in the Lindblad form, which is going to be of great use in the calculation of the heat current and work flux (power) in and out of our single-qubit system due to the interaction with the atomic beam. As given in [50] the heat current and power are defined using the coherent evolution Hamiltonian and the dissipators as

$$
\begin{gathered}
J_{i}=\left\langle\mathcal{L}_{i}^{*}\left(H_{\mathrm{tot}}\right)\right\rangle=\operatorname{Tr}\left[\rho_{q} \mathcal{L}_{i}^{*}\left(H_{\mathrm{tot}}\right)\right], \\
P=\left\langle\frac{\partial H_{\mathrm{tot}}}{\partial t}\right\rangle=\operatorname{Tr}\left[\rho_{q} \frac{\partial H_{\mathrm{tot}}}{\partial t}\right] .
\end{gathered}
$$

Here they are defined in the laboratory frame; however, we calculate them in the interaction picture for convenience. Using $U=e^{i \omega_{q} \sigma_{z} t / 2}$,

$$
\begin{aligned}
J_{i} & =\operatorname{Tr}\left[\rho_{q} \mathcal{L}_{i}^{*}\left(H_{\mathrm{tot}}\right)\right]=\operatorname{Tr}\left[U \rho_{q} \mathcal{L}_{i}^{*}\left(H_{\mathrm{tot}}\right) U^{\dagger}\right] \\
& =\operatorname{Tr}\left[U \rho_{q} U^{\dagger} U \mathcal{L}_{i}^{*}\left(H_{\mathrm{tot}}\right) U^{\dagger}\right]=\operatorname{Tr}\left[\tilde{\rho_{q}} \tilde{\mathcal{L}}_{i}^{*}\left(U H_{\mathrm{tot}} U^{\dagger}\right)\right],
\end{aligned}
$$

where the tilde denotes interaction picture terms. Since the Hamiltonian in the interaction picture [Eq. (7)] is given as

$$
H_{\mathrm{eff}}=U H_{\mathrm{tot}} U^{\dagger}-i U \frac{\partial U^{\dagger}}{\partial t},
$$

the heat current is obtained as

$$
J_{i}=\operatorname{Tr}\left[\tilde{\rho_{q}} \tilde{\mathcal{L}}_{i}^{*}\left(H_{\mathrm{eff}}+\frac{1}{2} \omega_{q} \sigma_{z}\right)\right] .
$$

Dropping the tilde for convenience and denoting $\left\langle\sigma_{z}\right\rangle=\rho_{e e}-$ $\rho_{g g},\left\langle\sigma_{+}\right\rangle=\rho_{g e}$, we calculate the heat current by directly using the definition of Eq. (17) as

$$
\begin{aligned}
J_{q}= & J_{1}+J_{2} \\
= & \frac{\gamma}{4} \omega_{q}\left(1-(2 N+1)\left\langle\sigma_{z}\right\rangle\right) \\
& -\frac{\gamma}{2} \operatorname{pg} \tau\left[\lambda^{*}\left(\frac{2 N+1}{2}\left\langle\sigma_{-}\right\rangle+\left\langle\sigma_{+}\right\rangle M\right)\right. \\
& \left.+\lambda\left(\left\langle\sigma_{-}\right\rangle M^{*}+\frac{2 N+1}{2}\left\langle\sigma_{+}\right\rangle\right)\right] .
\end{aligned}
$$

We can see from the above equation that $M$ can contribute heat flow only when $\lambda \neq 0$, which leads to a nonthermal working qubit state. In other words, if $\lambda=0, M$ cannot influence the working qubit populations. Accordingly, $M$ consists of ineffective coherence that cannot be regarded as heat-exchange coherences. Only those coherences in $N$ can contribute to heat flow and temperature of the work qubit under the condition that the qubit is described by the canonical thermal state in equilibrium.

Let us now calculate the power. Using Eq. (14) in the interaction picture, similar to heat current, we calculate the power to be

$$
P=\frac{1}{2} \hbar \dot{\omega}_{q}\left\langle\sigma_{z}\right\rangle+\operatorname{pg} \tau\left(\dot{\lambda}\left\langle\sigma_{+}\right\rangle+\dot{\lambda}^{*}\left\langle\sigma_{-}\right\rangle\right) .
$$

In order to find the explicit forms of the heat current and power, we need to solve the Bloch equations. Their steadystate solutions are presented in Appendix C. However, one can reach quick conclusions by looking at the above expressions for heat current and power. The most important one is that when there is no coherent drive in the system there is no power received by the system qubit from the external source. Heat-exchange coherences, the ones that are generated by the collective interaction of the atomic pairs with a heat bath, only contribute to the heat flux into the qubit system through the interactions with the atomic beam. As we see next, this heat flux eventually leads to a change in the thermal state of the single qubit.

The coherences that can be generated by the collective coupling of the atomic pair to the heat reservoirs lead to $\lambda=0$ and $\epsilon=0$. Specifically, only $a_{23}$ and $a_{32}$ can be generated 


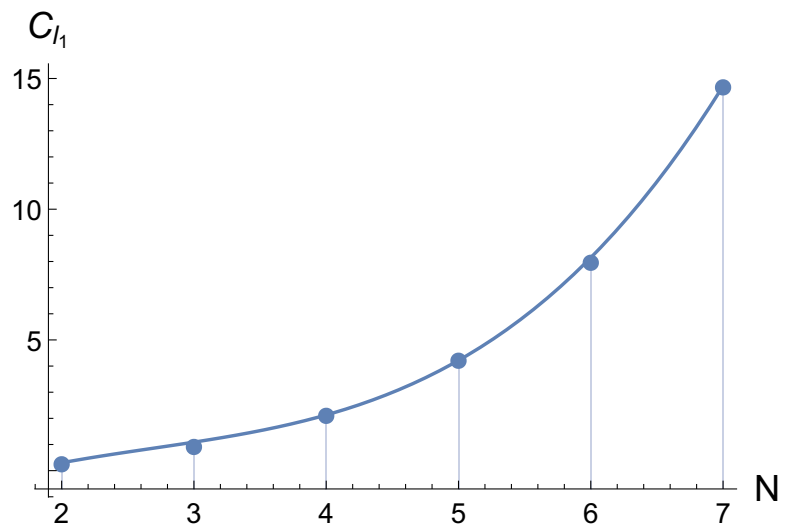

FIG. 4. Scaling of $l_{1}$ norm of coherence in the long-time limit with the number of atoms with an initial state where all the atoms are in their initial state. The dots represent the actual value of the coherence measure while the solid line is the cubic fit to these points. Model parameters are set to $f_{0} / \gamma_{0}=1$ and $n=10$.

(assuming initially the pair has no other coherence or no coherence at all). The master equation then becomes

$$
\dot{\rho}_{q}=\mu\left(\frac{r_{e}}{2} \mathcal{L}_{e} \rho_{q}+\frac{r_{d}}{2} \mathcal{L}_{d} \rho_{q}\right)
$$

This equation can be solved exactly and the steady-state solution of the density matrix is given by

$$
\rho_{s s}=\left(\begin{array}{cc}
\frac{r_{e}}{r_{d}+r_{e}} & 0 \\
0 & \frac{r_{d}}{r_{d}+r_{e}}
\end{array}\right) .
$$

We can assign an effective temperature for the atom as $T=-\left(\hbar \omega_{0} / k_{b}\right) \ln \left(r_{e} / r_{d}\right)$. Depending on $r_{e}$ and $r_{d}$ or the populations and coherences $a_{23}, a_{32}$ of the atomic pairs, the effective temperature can be negative or positive. At negative temperature, population inversion occurs. For $r_{e}<r_{d}$ a well-defined temperature exists and depends on coherences of the atomic pairs. In such a case, we conclude that thermally produced coherences are harvested back again as heat. The combination of collective heating and collisional harvesting schemes allows for exchange between quantum coherences and heat energy.

\section{LONG-TIME BEHAVIOR FOR DIFFERENT-SIZED ENSEMBLES}

We consider the case of initiating all atoms in our ensemble in their ground state, i.e., $\rho(t=0)=$ $\left|g_{1} g_{2} \cdots g_{N}\right\rangle\left\langle\left|g_{1} g_{2} \cdots g_{N}\right|\right.$. Such a state has no coherence in our reference basis. However, looking at the dynamics of $C_{l_{1}}$ we observe that it increases monotonically with time and settles to a finite value in the long-time limit, $C_{l_{1}}^{\text {lt }}$. The amount of coherence that is accumulated in the system increases with the increasing number of atoms in the ensemble; to be specific it shows a cubic best-fit behavior, as presented in Fig. 4. The values of $f_{0}$ and $\gamma_{0}$ have a negligible effect on the value that the coherence settles; however, they can affect the time it takes to reach this particular value. $C_{l_{1}}^{\text {lt }}$ is only controlled by $\bar{n}$. It grows from zero with increasing $\bar{n}$ up to a certain value and than saturates.
The off-diagonal density matrix elements that contribute to the nonzero value of $C_{l_{1}}$ are the ones in the block diagonals adjacent to the main diagonal. To be more specific, if we group our reference basis according to the number of excitations, only matrix elements that are inside the symmetric subspaces of these groups have a finite value. These blocks are also known as the Jordan-Wigner blocks of a density matrix with a given basis. The smallest system of a pair of atoms has a state in the form of a symmetric $X$-type state structure with real positive coherences. Larger ensembles still possess only real positive coherences in the blocks along the main diagonal. In [32], it was shown that the $\rho_{i j}$ 's inside these blocks have a caloric value and are called heat-exchange coherences. This implies that, for example, when the atomic cluster is injected in a cavity, these coherences will change the temperature of the cavity and thermalize it to a different finite value. Their contribution to the thermalization temperature is given by the addition of the coherences in Dicke-type blocks. For real positive coherences the coherence measure $C_{l_{1}}$ then directly characterizes the caloric value of harvesting such coherences in micromaser-type photonic quantum heat engines.

The initial state of the system that we consider in this section has no energy cost in the state-preparation stage since all atoms are initiated in their ground state. The energy cost of generating coherences is paid by the thermal drive. If this stage can be done by utilizing natural thermal resources, that can lead to reduced operational cost and hence increased efficiency of a quantum thermal machine.

\section{CONCLUSION}

We have investigated the dynamics and the long-time behavior of coherence in an atomic cluster that has dipolar interactions between the atoms, and is also subject to dissipation and driving by the thermal photons in the environment. We began our discussion by trying to understand the effects of dipole-dipole interaction strength and the mean number of environmental photons on the dynamics of coherence. In order to present the explicit results analytically, we first consider a pair of atoms, then explore larger clusters, up to seven atoms, numerically.

In the absence of thermal photons, $\bar{n}$, there is only dissipation on the atoms due to spontaneous emission processes whose rate is given by $\gamma_{0}$. Therefore, no coherence is generated nor does the present coherence survive in the joint state of the atoms over the course of the dynamics. In the case of a finite-temperature environment, where $\bar{n} \neq 0$, the system is driven by the photons, making it possible to generate and/or preserve the coherence. However, the very presence of photons also induces thermal emission processes, which creates a trade-off between dissipation and drive mechanisms. As a result the generated coherence cannot increase indefinitely and is not affected by increasing $\bar{n}$ after a certain number.

The dipolar interactions have an impact on some of the initial states, but not all. When the time evolution is started from one of these states, coherence showed an oscillator behavior in short times and it settled to the value determined by the dissipation and drive terms. In other words, the long-time value of coherence is indifferent to the dipole interactions. 
Moreover, the interaction strength $f_{0}$ must be greater than the $\bar{n} \gamma_{0}$ to be able to have this effect on the system in short times.

Furthermore, we have calculated the scaling behavior of coherence in the long-time limit for atomic clusters up to $N=7$. We chose to initiate all atoms in their ground state, for which there is no coherence present in the system. However, during the dynamics a certain amount of coherence builds up in the cluster, saturates, and becomes time invariant for the rest of the evolution. Coherence is created in the ensemble due to the fact that atoms are considered to be very close to each other. An excitation created by absorption of a photon, which is made possible by the drive mechanism, delocalizes throughout the system and puts it in a superposition state of all single-excitation subspace. Keeping in mind the losses in the system, coherence cannot grow to its maximum, but gain and loss balance each other to leave the system in a state with finite coherence.

We would like to emphasize that the creation and preservation of coherence in the model considered here has its explanation in the collective quantum effects taking place in our atomic cluster due to their proximity. Other explanations of enhanced coherence lifetime such as non-Markovianity [13-16] or decoherence-free subspaces [51] are not the case for our model. The former requires negative decay rates, $\gamma_{i j}<0$, to be present and the latter shows up in the pure dephasing dynamics of the subject system; both are features that our system does not possess.

Fundamentally we presented the heat equivalent of certain quantum coherences in atomic clusters by identifying means of mutual exchange between heat and quantum coherences. Accordingly the motive power of quantum coherence can be defined for the operation of quantum machines between quantum reservoirs with a coherence gradient. Our results can also be practically significant for reducing operational costs of quantum heat engines by utilizing natural quantum coherence resources and for designing multiqubit quantum machines where both the resource and working fluid parts consists of qubits, reducing the interfacing challenges. Searching for the existence of such mechanisms in biological systems or utilizing them for photovoltaic applications can be envisioned.

\section{ACKNOWLEDGMENTS}

The authors acknowledge support from a University Research Agreement between Lockheed-Martin Corp. and Koç University. The authors would like to thank J. Anders, M. Esposito, and M. Campisi for fruitful discussions.

\section{APPENDIX A: EFFECT OF DIPOLAR INTERACTIONS}

When only coherent dipole-dipole interaction is present, i.e., in the absence of drive and dissipation which corresponds to the $\gamma_{0}=0$ case, the elements of the density matrix of a two-atom ensemble evolves as follows:

$$
\begin{aligned}
& \rho_{11}(t)=\rho_{11}(0), \\
& \rho_{12}(t)=\rho_{12}(0) \cos (f t)+i \rho_{13}(0) \sin (f t), \\
& \rho_{13}(t)=\rho_{13}(0) \cos (f t)+i \rho_{12}(0) \sin (f t), \\
& \rho_{14}(t)=\rho_{14}(0),
\end{aligned}
$$

$$
\begin{aligned}
\rho_{22}(t)= & {\left[\left(\rho_{22}(0)+\rho_{33}(0)\right)+\left(\rho_{22}(0)-\rho_{33}(0)\right) \cos (2 f t)\right.} \\
& \left.+2 i \operatorname{Im}\left[\rho_{23}\right] \sin (2 f t)\right] / 2, \\
\rho_{23}(t)= & {\left[\operatorname{Re}\left[\rho_{23}(0)\right]+i\left(2 \operatorname{Im}\left[\rho_{23}(0)\right] \cos (2 f t)+\left(\rho_{22}(0)\right.\right.\right.} \\
& \left.\left.\left.-\rho_{33}(0)\right) \sin (2 f t)\right)\right] / 2, \\
\rho_{24}(t)= & \rho_{24}(0) \cos (f t)-i \rho_{34}(0) \sin (f t), \\
\rho_{33}(t)= & {\left[\left(\rho_{22}(0)+\rho_{33}(0)\right)-\left(\rho_{22}(0)-\rho_{33}(0)\right) \cos (2 f t)\right.} \\
& \left.-2 i \operatorname{Im}\left[\rho_{23}(0)\right] \sin (2 f t)\right] / 2, \\
\rho_{34}(t)= & \rho_{34}(0) \cos (f t)-i \rho_{24}(0) \sin (f t), \\
\rho_{44}(t)= & \rho_{44}(0),
\end{aligned}
$$

where $\rho_{i j}(0)$ 's are their initial values. By looking at the above equations, we can determine which initial states will not be affected by the dipolar interactions over the course of time. One can immediately see that states residing in the $\rho_{11}, \rho_{14}$ (and naturally $\rho_{41}$ which is $\rho_{14}^{*}$ ), and $\rho_{44}$ subspace initially do not feel the presence of dipole-dipole interaction. Moreover, states having $\rho_{22}(0)=\rho_{33}(0)$ and $\rho_{23}(0) \in \operatorname{Re}$ will also be indifferent to the dipolar interactions.

\section{APPENDIX B: TIME-EVOLUTION OPERATOR}

The time-evolution operator is given as follows:

$U(\tau)=\exp \left(-i H_{\mathrm{int}} \tau\right)=\exp \left(-i g \tau \sum_{i=1}^{2}\left(\sigma_{i}^{+}{\sigma_{0}^{-}}^{-}+{\sigma_{i}^{-}}^{-} \sigma_{0}^{+}\right)\right)$,

where we took $\hbar=1$. We need to evaluate this operator up to second order in $(g \tau)^{2}$. Thus if we set $S=\sum_{i=1}^{2}\left(\sigma_{i}^{+} \sigma_{0}^{-}+\sigma_{i}^{-} \sigma_{0}^{+}\right)$, the evolution operator to second order in $(g \tau)^{2}$ is

$$
U(\tau) \approx \mathbb{1}-i g \tau S-\frac{(g \tau)^{2}}{2} S^{2}
$$

The collective raising and lowering operators are given as

$$
\begin{aligned}
& \sum_{i=1}^{2} \sigma_{i}^{+}=\left(\begin{array}{llll}
0 & 1 & 1 & 0 \\
0 & 0 & 0 & 1 \\
0 & 0 & 0 & 1 \\
0 & 0 & 0 & 0 \\
& & &
\end{array}\right), \\
& \sum_{i=1}^{2} \sigma_{i}^{-}=\left(\begin{array}{llll}
0 & 0 & 0 & 0 \\
1 & 0 & 0 & 0 \\
1 & 0 & 0 & 0 \\
0 & 1 & 1 & 0
\end{array}\right),
\end{aligned}
$$

which give us the $S$ operator in the following form:

$$
S=\left(\begin{array}{cccc}
0 & \sigma_{0}^{-} & \sigma_{0}^{-} & 0 \\
\sigma_{0}^{+} & 0 & 0 & \sigma_{0}^{-} \\
\sigma_{0}^{+} & 0 & 0 & \sigma_{0}^{-} \\
0 & \sigma_{0}^{+} & \sigma_{0}^{+} & 0
\end{array}\right) .
$$


Finally the time-evolution operator is evaluated as

$$
U(\tau)=\left(\begin{array}{cccc}
1-(g \tau)^{2} \sigma_{0}^{-} \sigma_{0}^{+} & -i g \tau \sigma_{0}^{-} & -i g \tau \sigma_{0}^{-} & 0 \\
-i g \tau \sigma_{0}^{+} & 1-(g \tau)^{2} / 2 & -(g \tau)^{2} / 2 & -i g \tau \sigma_{0}^{-} \\
-i g \tau \sigma_{0}^{+} & -(g \tau)^{2} / 2 & 1-(g \tau)^{2} / 2 & -i g \tau \sigma_{0}^{-} \\
0 & -i g \tau \sigma_{0}^{+} & -i g \tau \sigma_{0}^{+} & 1-(g \tau)^{2} \sigma_{0}^{+} \sigma_{0}^{-}
\end{array}\right) .
$$

\section{APPENDIX C: STEADY-STATE SOLUTIONS OF THE BLOCH EQUATIONS}

The steady-state solutions of Eqs. (19) are found as follows:

$$
\begin{aligned}
& \left\langle\sigma_{x}\right\rangle_{s s}=\frac{\frac{i \gamma p g \tau}{4}\left[\left(2 N+M+M^{*}+1\right)\left(\lambda-\lambda^{*}\right)+\left(M-M^{*}\right)\left(\lambda+\lambda^{*}\right)\right]}{d}, \\
& \left\langle\sigma_{y}\right\rangle_{s s}=\frac{\frac{-\gamma p g \tau}{4}\left[\left(2 N+M+M^{*}+1\right)\left(\lambda+\lambda^{*}\right)-\left(M-M^{*}\right)\left(\lambda-\lambda^{*}\right)\right]}{d}, \\
& \left\langle\sigma_{z}\right\rangle_{s s}=\frac{\frac{\gamma^{2}}{4}\left[\left(2 N+M+M^{*}+1\right)^{2}+\left(M-M^{*}\right)^{2}\right]}{d},
\end{aligned}
$$

where $d$ is given as

$$
d=\left[(p g \tau)^{2}\left(\left(M-M^{*}\right)\left(\lambda^{2}-\lambda^{* 2}\right)-2|\lambda|^{2}\left(2 N+M+M^{*}+1\right)\right)-\frac{\gamma^{2}(2 N+1)}{4}\left(\left(2 N+M+M^{*}+1\right)^{2}+\left(M-M^{*}\right)^{2}\right)\right] .
$$

[1] H.-P. Breuer and F. Petruccione, The Theory of Open Quantum Systems (Oxford University Press, Oxford, 2007).

[2] R. Alicki and K. Lendi, Quantum Dynamical Semigroups and Applications (Springer, Berlin, 2007).

[3] Á. Rivas and S. F. Huelga, Open Quantum Systems, An Intorduction (Springer, Heidelberg, 2012).

[4] T. Baumgratz, M. Cramer, and M. B. Plenio, Phys. Rev. Lett. 113, 140401 (2014).

[5] F. Levi and F. Mintert, New J. Phys. 16, 033007 (2017).

[6] D. Girolami, Phys. Rev. Lett. 113, 170401 (2014).

[7] I. Marvian and R. W. Spekkens, Phys. Rev. A 94, 052324 (2016).

[8] G. Karpat, B. Çakmak, and F. F. Fanchini, Phys. Rev. B 90, 104431 (2014).

[9] A. L. Malvezzi, G. Karpat, B. Çakmak, F. F. Fanchini, T. Debarba, and R. O. Vianna, Phys. Rev. B 93, 184428 (2016).

[10] T. R. Bromley, M. Cianciaruso, and G. Adesso, Phys. Rev. Lett. 114, 210401 (2015).

[11] S. Bhattacharya, S. Banerjee, and A. K. Pati, arXiv:1601.04742.

[12] F. Damanet and J. Martin, J. Phys. B 49, 225501 (2016).

[13] Y.-N. Guo, K. Zeng, Q.-L. Tian, and Z.-D. Li, arXiv:1612.08791.

[14] C. Addis, G. Brebner, P. Haikka, and S. Maniscalco, Phys. Rev. A 89, 024101 (2014).

[15] Y.-J. Zhang, W. Han, Y.-X. Xia, Y.-M. Yu, and H. Fan, Sci. Rep. 5, 13359 (2015).

[16] N. Behzadi, B. Ahansaz, and E. Faizi, arXiv:1607.06507.

[17] A. Streltsov, G. Adesso, and M. B. Plenio, arXiv:1609.02439 [Rev. Mod. Phys. (to be published)].
[18] J. I. de Vicente and A. Streltsov, J. Phys. A 50, 045301 (2017).

[19] A. Streltsov, H. Kampermann, S. Wölk, M. Gessner, and D. Bruss, arXiv:1612.07570.

[20] S. Rana, P. Parashar, A. Winter, and M. Lewenstein, arXiv:1612.09234.

[21] Y. Yao, X. Xiao, L. Ge, and C. P. Sun, Phys. Rev. A 92, 022112 (2015).

[22] A. Streltsov, U. Singh, H. S. Dhar, M. N. Bera, and G. Adesso, Phys. Rev. Lett. 115, 020403 (2015).

[23] K. Brandner and U. Seifert, Phys. Rev. E 93, 062134 (2016).

[24] K. Brandner and U. Seifert, arXiv:1703.02464.

[25] R. Uzdin, Phys. Rev. Appl. 6, 024004 (2016).

[26] S. F. Huelga and M. B. Plenio, Contemp. Phys. 54, 181 (2013).

[27] N. Lambert, Y.-N. Chen, Y.-C. Cheng, C.-M. Li, G.-Y. Chen, and F. Nori, Nat. Phys. 9, 10 (2012).

[28] G. D. Scholes, G. R. Fleming, A. Olaya-Castro, and R. van Grodelle, Nat. Chem. 3, 763 (2011).

[29] H.-B. Chen, J.-Y. Lien, C.-C. Hwang, and Y.-N. Chen, Phys. Rev. E 89, 042147 (2014).

[30] M. O. Scully, M. S. Zubairy, G. S. Agarwal, and H. Walther, Science 299, 862 (2003).

[31] C. A. Rodriguez-Rosario, T. Frauenheim, and A. Aspuru-Guzik, arXiv:1308.1245.

[32] C. B. Dağ, W. Niedenzu, Ö. E. Müstecaplıoğlu, and G. Kurizki, Entropy 18, 244 (2016).

[33] D. Türkpençe and Ö. E. Müstecaplıoğlu, Phys. Rev. E 93, 012145 (2016). 
[34] J. Åberg, Phys. Rev. Lett. 113, 150402 (2014).

[35] M. Lostaglio, D. Jennings, and T. Rudolph, Nat. Commun. 6, 6383 (2015).

[36] K. Korzekwa, M. Lostaglio, J. Oppenheim, and D. Jennings, New J. Phys. 18, 023045 (2016).

[37] S. Schneider and G. J. Milburn, Phys. Rev. A 65, 042107 (2002).

[38] F. Benatti, R. Floreanini, and M. Piani, Phys. Rev. Lett. 91, 070402 (2003).

[39] V. Vedral, New J. Phys. 6, 102 (2004).

[40] T. Choi and H.-J. Lee, Phys. Rev. A 76, 012308 (2007).

[41] Z. Ficek and R. Tanaś, Phys. Rep. 372, 369 (2002).

[42] J. B. Brask, G. Haack, N. Brunner, and M. Huber, New J. Phys. 17, 113029 (2015).
[43] V. Scarani, M. Ziman, P. Štelmachovič, N. Gisin, and V. Bužek, Phys. Rev. Lett. 88, 097905 (2002).

[44] M. Ziman, P. Štelmachovič, V. Bužek, M. Hillery, V. Scarani, and N. Gisin, Phys. Rev. A 65, 042105 (2002).

[45] G. S. Agarwal, Quantum Statistical Theories of Spontaneous Emission and Their Relation to Other Approaches (Springer, Berlin, 1974).

[46] M. J. Stephen, J. Chem. Phys. 40, 669 (1964).

[47] R. H. Lehmberg, Phys. Rev. A 2, 883 (1970).

[48] C. W. Gardiner, Phys. Rev. Lett. 56, 1917 (1986).

[49] S. Banerjee and R. Srikanth, Eur. Phys. J. D 46, 335 (2008).

[50] R. Kosloff, Entropy 15, 2100 (2013).

[51] D. A. Lidar, Adv. Chem. Phys. 154, 295 (2014). 